\title{
Suppression of running times by olfactory stimuli'
}

ROBERT J. COURTNEY, Jr., LARRY D. REID, and RONALD E. WASDEN, Department of Psycholog!, Bradley University Peoria, Ill. 61606

Thirsty rats running a straight-way for water were exposed to the odor of rats which had received foot-shock, a perfumed spray deodorant, and the essence of cat. Running times of Ss increased when they first encountered each of the odors. The odor of cat was particularly effective in suppressing Ss' approach. The first encounter with the odors seemed to function as a mild punishment. Control for odors such as those left by rats which have received foot-shock may be important in many experimental situations.

While investigating approach-avoidance behavior in a straightway, it was observed that running times were conspicuously greater on the day rats were to be given foot-shock compared to their previous trials, even though they had not yet been shocked. Although no mention of this phenomenon could be found in a search of the literature, it was surmised that Ss perceived olfactory cues left by the previously shocked rats.

Olfactory cues have been found to be important in guiding the behavior of animals, including approach and avoidance behavior to similar and different species. For instance, in wild animals scent-marks may serve as territorial markers (Hediger, 1950). Wild rats use olfactory cues for distinguishing pack membership (Lorenz, 1966). In general, rats tend to avoid strange stimuli, but no similar tendency to avoid unfamiliar odors has been observed in laboratory rats (Bamett, 1963). Rats leave urine and genital secretions to which other rats are sensitive; however, in artificial colonies, it appears that such trails have an attractive effect (Reiff, 1952). More recently, it has been suggested that the influence of olfactory cues plays only a minor role in determining the amount of exploratory behavior of laboratory rats (Halliday, 1967). Since our observations were in apparent conflict with some of these conclusions, it was decided to investigate the potential suppressive effects of various odors more systematically.

\section{METHOD}

Nine, male, Holtzman rats, about 120 days old at the beginning of the experiment, were trained to run in an unpainted plywood straight-way that was $7 \mathrm{ft}$ long, $6 \mathrm{in}$. wide, and $11 \mathrm{in}$. deep. The goalend of the alley was marked by an 18-in. floor of spaced stainless steel bars and contained a retractable water tube. Time that elapsed from the opening of the door of the startbox until S touched the water spout, the dependent variable of this experiment, was automatically recorded. Foot-shock was delivered by the technique described by Flax \& Hahn (1967). The maze was washed with clear water every day about $3 \mathrm{~h}$ before Ss were tested.
Ss were allowed to drink for 20 min only once a day until they drank enough at a single daily opportunity to maintain their predeprivation weight. Ss were then trained to run the alley for the chance to drink for $3 \mathrm{sec}$ a trial. They were run, as they were on all subsequent days of testing, five trials a day with an intertrial interval of 20 sec. Twenty min after a daily session, Ss were given a 10-min opportunity to drink in their home cages. Ss were always tested in the same order.

Ss were trained until they all ran the maze in less than $6 \mathrm{sec}$, eight out of 10 trials. They were then further trained for seven daily sessions to establish baseline performances; their average running times (the mean of Ss' daily mean) are designated $\mathrm{C}_{1}$ through $\mathrm{C}_{7}$ in Fig. 1. These baseline performances are similar enough to conclude that they are samples drawn from the same population $(\mathrm{F}=.35, \mathrm{df}=8,54)$.

For two days following the determination of baselines, four extra rats received foot-shock in the goalbox $(2.5 \mathrm{~mA}$ until they escaped) before the experimental Ss were tested. These punished Ss ran to the start-end of the alley and were then pushed with a paddle toward the goal before they were removed. A rat was punished in this way before the first, second, fourth, and seventh experimental Ss were tested. This procedure was repeated on all days designated by an " $S$ " in Fig. 1 . On all subsequent days labeled with a " $\mathrm{C}$ " in Fig. 1 the shock generator was turned on and off and the paddle dragged through the maze to mimic the procedure of the previous days on which the "odor-producing rats" had been shocked.

On days labeled with a "B" in Fig. 1 Ban Spray Deodorant was sprayed generously throughout the alley at the same inter-S intervals as the "odor-producing rats" were used. On the 27 th day of testing (labeled "CAT" in Fig. 1), a male cat was placed in the alley and allowed to walk through it at the same inter-S intervals of previous tests. On the 39th day of testing all Ss were given foot-shock $(1.0 \mathrm{~mA}$ for $.5 \mathrm{sec})$ as they were drinking to further assess the relative suppressive effects of the various odors.

\section{RESULTS AND DISCUSSION}

There are obvious statistical differences among the various daily samples of running times, since the distribution of some of the samples do not overlap with the distribution of other samples. It was decided to compare by t-tests the overall mean of the daily running times of the first seven control days to means of various experimental days in order to summarize the magnitude of the differences. Since Ss' running times would in general be expected to improve with practice (compare means of first seven control days to $C_{11}$ through $C_{16}$ of Fig. 1), comparing subsequent experimental days with the first control days provides a conservative estimate of the reliability of potential differences being tested. Student ts of the daily running times of the first seven control days and of days of the first encounter with a new

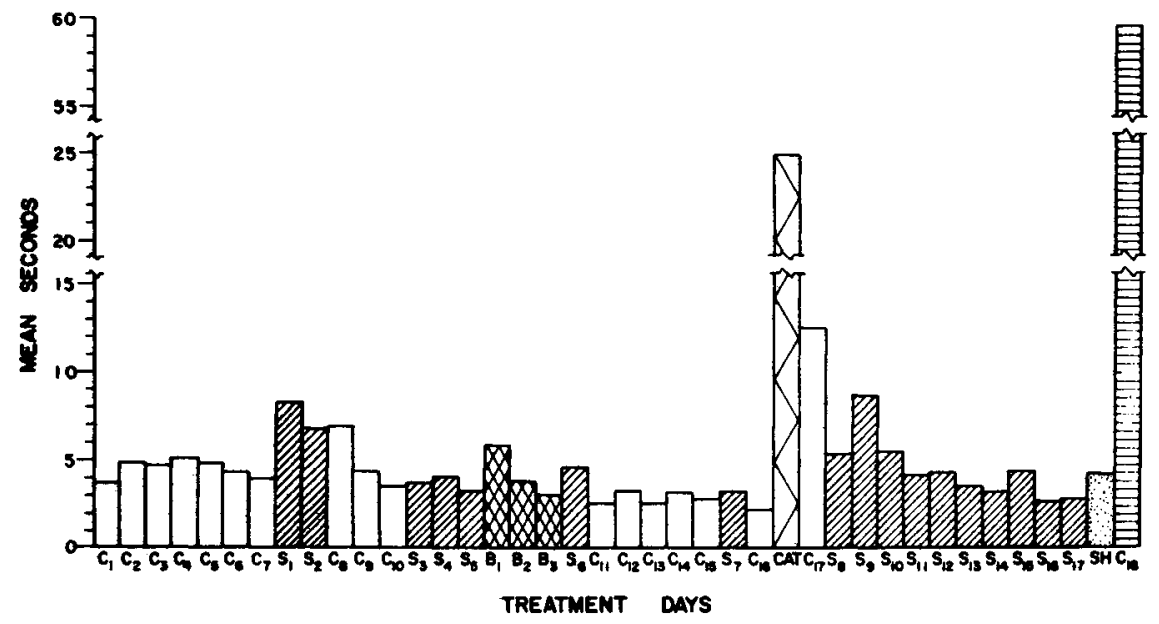

Psychon. Sci., 1968, Vol. 12 (7)
Fig. 1. Average running times of nine Ss for water reinforcement. Experimental treatments are given in order from left to right with each bar representing the mean of Ss' average daily running times. $\mathrm{C}=\mathrm{Control}$; $S=$ Odor of shocked rat; $B=$ Odor of Ban; SH = Day of foot-shock; CAT $=$ Odor of cat. 
odor are $3.9,1.7$, and $6.9(\mathrm{df}=70)$, respectively for the scores comparing odor of shocked-Ss, Ban, and cat. The greatest average increment in running times, aside from the trials following punishment itself, was on the day that the cat was placed in the straight-way; in fact three of the nine Ss took longer to run on the day of the cat-odor than they did following being punished by foot-shock. Two of the nine Ss had "runny noses" (mucus secretions at the nostrils) on every test day and, interestingly enough, were least affected by the presence of the odors.

The animal odors produced more of a suppression of running times than did the perfumed spray deodorant; consequently, we conclude that mere novelty of odor is not a complete explanation of the Ss' hesitancy to run and that animal odors are stimuli that have high impact for rats. Had Halliday (1967) used animal odors such as used here, he might have found that odors are important determiners of amount of exploration among rats.

The Ss' approach was most suppressed on the first trials of the day in which they encountered the new odors, e.g., when the first trials of the first seven control days were compared to the first trials of the day when Ss were first exposed to the odor of shock-rats, it was found that average running times were tripled (mean of control $=6.3 \mathrm{sec}$, mean of day of odor $=21.7 \mathrm{sec}$, $t=2.3, \mathrm{df}=70$ ). The odor of the deodorant was quickly habituated to, and by the second day of exposure, Ss ran as fast as they did on the first control days.

Ss habituated to the odor of shocked-Ss across days as well as within a day's trials. Thus, shocking nonexperimental Ss before Ss are run during pretraining and before $S s$ are to be shocked themselves should avoid the potential confounding that might occur from the interaction between the suppressing effect of the odor of a previously shocked $S$ and the suppressing effect of foot-shock. In a subsequent approach-avoidance experiment (Courtney, 1968) it was found that Ss did not run slowly on the day they received shock as had our Ss of other previous studies (also compare the average running of the day $S s$ received foot-shock, labeled "SH" in Fig. 1 to control days).

On the control days following the first introduction of the odor of shocked-Ss $\left(C_{8}\right)$ and the odor of cat $\left(C_{17}\right)$, Ss' approach was still suppressed (ts $=1.9$ and 3.2 , respectively, $\mathrm{df}=70$ ). It is not likely that the suppression on the day following the odor of cat was due to the lingering odor. After all Ss were run on the day of the cat, the straight-way was completely washed and Ss from another experiment were run in the maze about $4 \mathrm{~h}$ later. These $\mathrm{Ss}$ evidenced no increased running times. It is likely that the continued suppression of running times, noted on control days following the introduction of animal odors, is a result of conditioning of the "fear" of these odors to maze-running (Pain \& Booth, 1968).

An analysis of variance of scores of all sessions except those compared by t-test to the first seven control days and the scores of days labeled " $\mathrm{S}_{2}$ " and " $\mathrm{C}_{18}$ " of Fig. 1 indicated that a further search for reliable differences was not warranted $(F=1.4$, $\mathrm{df}=25,208$ ).

It is highly adaptive for animals to fear without having to directly experience structural damage. Rats, even if they are highly deprived and approaching a familiar goal, are more apt to survive if they are cautious in places where other rats have been hurt or where potential predators have recently traveled.

\section{REFERENCES}

BARNETT, S. A. The rat: A study in behavior. Chicago: Aldine, 1963.

COURTNEY, R. J., Jr. Studies of approach-avoidance behavior. Unpublished Master's thesis, Bradley Univer., 1968.

FLAX, S. W., \& HAHN, W. H. Solid state shock "scrambler." American Journal of Psychology, 1967, 70, 446-447.

HALLIDAY, M. S. The influence of olfactory cues on exploratory behavior. Psychonomic Science, 1967, 9, 595-596.

HEDIGER, H. Wild animals in captivity. London: Butterworth, 1950.

LORENZ, K. On aggression. New York: Harcourt, Brace, \& World, 1966.

PAIN, J. F., \& BOOTH, D. A. Toxiphobia for odors. Psychonomic Science, $1968,10,363-364$.

REIFF, M. Über territorieumsmarkienung bei hausratten und hausmausen. Verh. Schweiz. Naturf. Ges. Luzern, 1952, 150-151. NOTE

1. This work was supported by funds made available by the Bradley University Faculty Research Committee to L. D. R. and R. E.W. 